\title{
An unexplained three-dimensional percept emerging from a bundle of lines
}

\author{
Eric L. Altschuler ${ }^{1}$ Abigail E. Huang ${ }^{2} \cdot$ Hee J. $\mathrm{Kim}^{3} \cdot$ Luca Battaglini $^{4}$ • \\ Sergio Roncato ${ }^{4}$
}

Published online: 6 July 2017

(C) The Psychonomic Society, Inc. 2017

\begin{abstract}
Perceptual grouping has been extensively studied, but some areas are still unexplored-in particular, the figural organizations that emerge when bundles of intersecting lines are drawn. Here, we will describe some figural organizations that emerge after the superimposition of bundles of lines forming the profile of regular triangular waves. By manipulating the lines' jaggedness and junction geometry (regular or irregular X junction) we could generate the following organizations: (a) a grid, or a figural configuration in which both the lines and closed contours are perceived, (b) a figure-ground organization composed of figures separated by portions of the background, and (c) a corrugated surface appearing as a multifaceted polyhedral shell crossed by ridges and valleys. An experiment was conducted with the aim at testing the role of the good-continuation and closure Gestalt factors. Good continuation prevails when the lines are straight or close to straightness, but its role is questionable in the appearance of a corrugated surface. This perceptual organization occurs
\end{abstract}

Electronic supplementary material The online version of this article (doi:10.3758/s13414-017-1369-7) contains supplementary material, which is available to authorized users.

Eric L. Altschuler

altschue@nychhc.org

Sergio Roncato

sergio.roncato@unipd.it

1 Department of Physical Medicine and Rehabilitation, Metropolitan Hospital, 1901 First Avenue, New York, NY 10029, USA

2 Department of Ophthalmology, Duke University School of Medicine, Durham, NC, USA

3 School of Medicine, Rutgers New Jersey Medical School, Newark, NJ, USA

4 Dipartimento di Psicologia Generale, Università di Padova, via Venezia 8, 35131 Padova, Italy despite the violation of the good-continuation rule and consists of a structure of such complexity so as to challenge algorithms of computer vision and stimulate a deeper understanding of the perceptual interpretation of groups of lines.

Keywords 3D perception: *Other $\cdot 2 \mathrm{D}$ shape and form

Perceptual grouping manifests when simple figural units appear to organize into a group distinct from the simple figural units. Several factors have been recognized to play a role in the formation of groups (Wertheimer, 1923; Wertheimer \& Spillmann, 2012; Wagemans et al., 2012).

We have an incomplete understanding of the properties that are associated with this appearance. A group of elements, such as dots, is not only a set of "figures that are perceived together" but is also a portion of the perceptual world that possesses an internal organization and the ability to condition other forms of organization (Pinna, 2010).

This organization may be very simple, for example, when it manifests as straightness (Beck, Rosenfeld, \& Ivry, 1989; Smits, Vos, \& Van Oeffelen, 1985, Uttal, 1973; Uttal, Bunnell, \& Corwin, 1970) or curvilinearity (Feldman, 1996, 1997). However, more complex structural attributes can be detected - for example, mirror symmetry (van der Helm, 2014; Wagemans, 1993), or a solid volume or a biological motion when dots are put in movement (Johansson, 1975).

Rich figural organizations arise when grouping factors act in synergy; for example, Glass (Glass \&Perez, 1973) and Marroquin (1976) patterns formed from collections of dots (points). When lines are used as the basic figural element these effects can be particularly difficult to explore due to the variability of parameters that come into play when we draw a line: length, orientation, straightness, and parallelism. 
If we draw two bundles of straight parallel lines, interesting phenomena do not emerge, since what we see is a grid or a tartan: The lines are clearly distinguishable as well as the rectangles they form (see Fig. 1). However, more complex figural organizations appear when the straight lines are replaced by waving or jagged lines. Fig. 2a provides a first example. Two bundles of jagged lines are superimposed; the surface they pass through appears to be divided in "tiles" (fused pentagons; see the image on the right). But look at Fig. 2b, where a bundle of lines has been laterally displaced. A new configuration appears in which star-like figures alternate with regular octagons. These configurations are the focus of enquiry of a branch of mathematics dealing with what are known as tilings and tessellations. Fig. $2 \mathrm{a}$ and $\mathrm{b}$ are two examples of tiling illustrated in the treatise on tiling and tessellation by Grünbaum and Shepard (1987). In effect, they correspond to the following definition: "A plane tiling $\mathrm{T}$ is a countable family of closed sets $\mathrm{T}=\{\mathrm{T} 1, \mathrm{~T} 2 \ldots\}$ which cover the plane without gaps or overlaps" (p. 16).

Figure $2 \mathrm{a}$ is an isohedral tiling, and Fig. $2 \mathrm{~b}$ a dihedral tiling. There are no gaps between the tiles (Grünbaum \& Shepard, 1987). Nevertheless the phenomenological observation demonstrates that the mathematical and the psychological descriptions diverge.

Phenomenological observations in Fig. 2a conform to the mathematical definition, since we perceive a surface filled, with no gaps, with identical figures similar to couples of fused pentagons - the prototile of the tiling.

A different percept arises in Fig. 2b. What we see is a series of stars arranged in regular rows and columns. They are against a background of star-like figures separated by a gap. But if considered as a dihedral tiling, Fig. $2 \mathrm{~b}$ is a surface segmented into stars and octagons with no interposed gap.

The different perceptual organizations emerging from Figs. $2 \mathrm{a}$ and $\mathrm{b}$ can be accounted for by the classic Gestalt grouping factors. Good continuation is responsible for the emergence of jagged lines as unitary percept (as it occurs

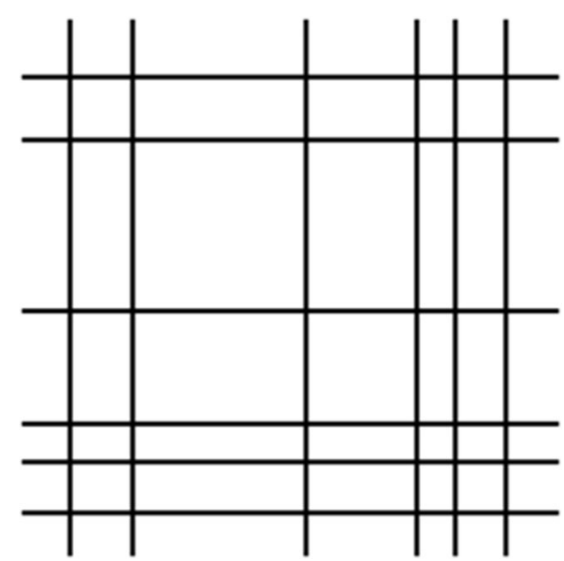

Fig. 1 Tartan pattern. Two identical bundles of lines orthogonally oriented when we perceive a grid or tartan-like figure); closure acts to make the closed contours emerge (see Fig. 2b). The balance of the two factors is likely to originate the perceptual coexistence of lines and closed contours - that is, the tiling we perceive in Fig. 2a.

A further set of demonstrations is obtained by acting on the orientation and the displacement of two bundles of lines. Again, the mathematical and the perceptual organizations sometimes diverge.

\section{Isohedral tiling}

Figure $2 \mathrm{c}$ is generated by two bundles of lines, one inclined by 60 degrees with respect the other. Identical tiles fill the whole surface, and the jagged lines are no longer distinguishable. Therefore, tiling corresponds to a perceptual as well as to a mathematical entity.

\section{Dihedral tiling}

In Fig. 2d, jagged lines intercepting at the flexion points form two prototiles: diamonds and squares. We perceive both shapes but these polygons are not perceptually coplanar. They appear as the facets of a regularly corrugated surface or a polyhedral surface. The jagged lines may be seen both as dividing lines of folded surfaces and as the sides of the facets.

\section{Trihedral tiling}

Figure 2e is the result of the superposition of bundles of lines with variable interspaces. There are three prototiles: a star, an octagon, and an eight-sided regular figure resulting from the merging of two pentagons (see the fused pentagons shape on the right). The perceptual organization is not a tiling, since we clearly distinguish an octagon and four star-like shapes, but not a third shape - that is, the fused pentagons - but rather these as a portion of the background. In other words, we perceive, instead of a tile, a gap or a fragment of a seamless surface partially hidden by an octagon and a four-star-like figure. The jagged lines therefore are seen to contour two shapes out of three, the third one appearing as a spatial gap interposed between them.

Interpreting these different perceptual organization as the result of the action of the classic grouping factors is nontrivial: Apparently, good continuation seems to prevail when we perceive the jagged lines in their whole extent, but the closure factor prevails when the jagged lines are no long seen and closed contours appear. When both the lines and the tiles are 
a

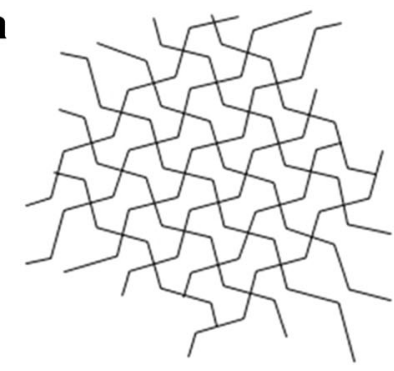

b

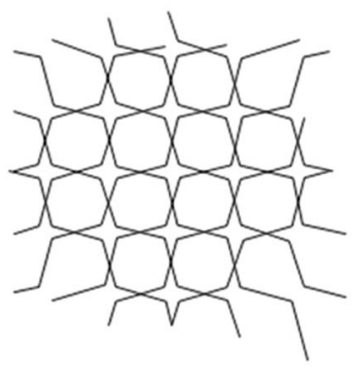

C

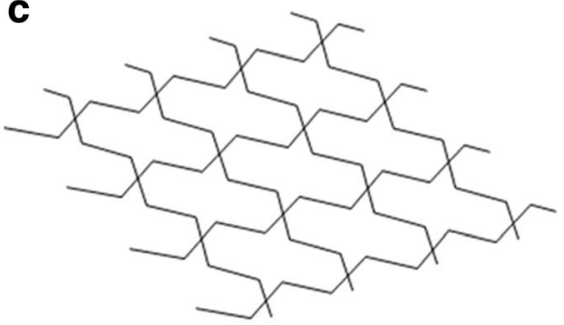

d

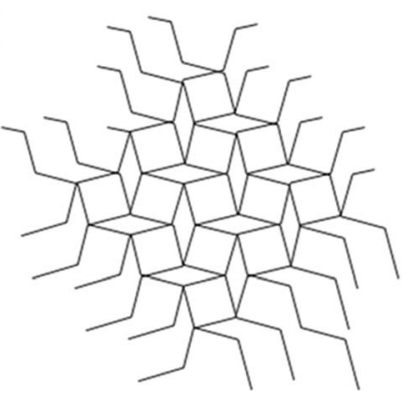

e

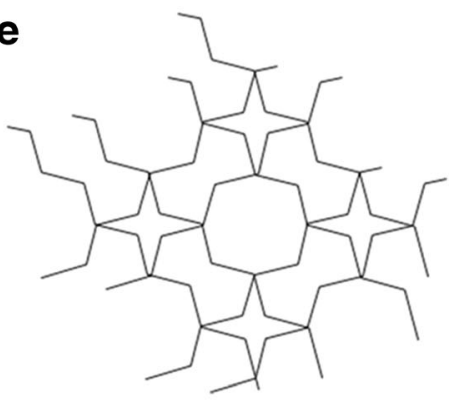

Fig. 2 Perceptual result of overlapping/superposing sets of jagged lines. The same jagged line is reused to obtain bundles in which the lines, like triangular waves, differ in phase. a The same bundles of lines regularly spaced and in opposition of phase. The lines are visible as well as the regular tiles they form (fused pentagons drawn in isolation on the right). $b$ Overlap of the same jagged lines as in a, drawn so as to obtain acute and obtuse angles at the intersection of the lines: a configuration of star-like shapes perceptually emerges. c More spaced jagged lines are organized in

perceived, we may hypothesize that a balance occurs so that the two solutions coexist.

Are these two factors sufficient to account for the rise of these perceptual organizations, or does some other structuring factor come into play?

In Figs. 3 and 4, a first demonstration is offered of the effects of straightness manipulation in configurations of jagged lines similar to Fig. $2 b$ and d.

\section{Line drawings, straightness, and spatial organization}

\section{Stars, octagons}

In Fig. 3, jagged lines similar to the ones employed in the previous demonstrations have been employed. The bundles of lines intercept so that regular X junctions form at the crossing points. From top down we can see the effects of the progressive lines straightening. Note that the star-like organization progressively vanishes. two intersecting bundles: a monotile configuration appears (i.e., a typical example of tiling). Note that the jagged lines are detectable after an attentive effort. $d$ Two bundles of jagged lines intersect at the inflection points giving the perception of a corrugated surface (two-dimensional nonflat manifold) in three-dimensional space. e The same jagged lines as in b periodically varying in interspaces. Two shapes are visible: a central octagon and four stars. Note that the contour of the interspaces separating the stars and the octagon forms has the fused-pentagons shape

The effects of varying the amount of jaggedness/ straightness are shown in the flanking portions of jagged lines. In Fig. 3a, the deviation is 66 degrees; in Fig. 3b, the deviation is 44 degrees; in Fig. 3c, 29 degrees. The upper figure (Fig. 3a) appears as a regular arrangement of stars. In the middle figure (Fig. 3b), successive straightening of the lines generates an ambiguous bistable percept: Sometimes we perceive stars and regular convex hexagons and sometimes crossing jagged lines. In the bottom figure (Fig. 3c) a further straightening makes the stars disappear and the jagged lines appear. (See Supplementary Material 1 for a more finely graded progression of straightening of the lines.)

\section{Diamonds, corrugation, orthogonal lines}

In Fig. 4, the pattern differs from the previous one because the jagged lines do not cross at the midpoint between two folding points. They are located so as to intercept at their folding points where they converge to form an irregular $\mathrm{X}$ junction. A portion of these jagged lines is drawn on the flanking space 


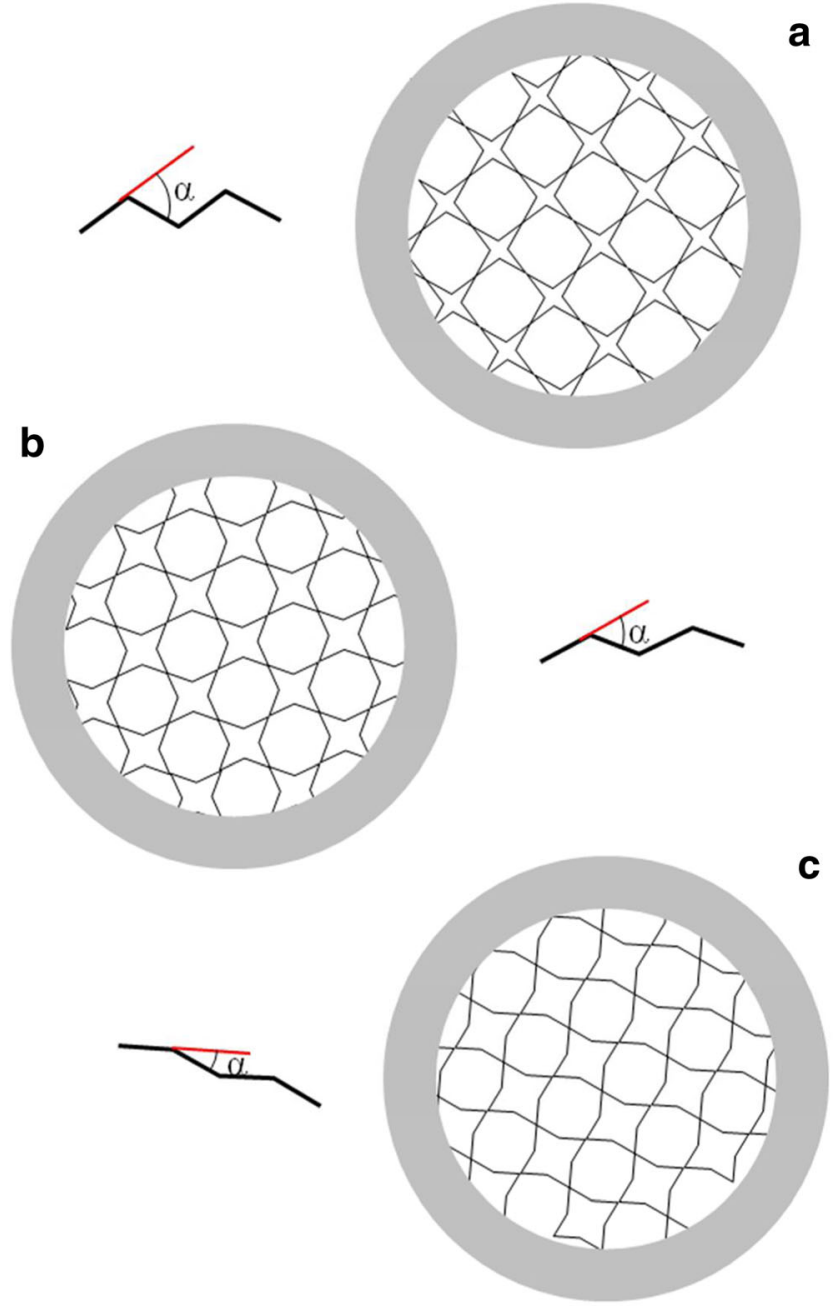

Fig. 3 Perceptual result of progressive straightening of jagged lines that cross at the midpoint between inflection points. Each figure is flanked by an enlargement of a portion of the figure to illustrate the deviation from straightness $(\alpha)$. See text for discussion. (Color figure online)

of Fig. 4 to illustrate the magnitude of the deviation from straightness.

In Fig. 4a, 76 degrees deviation $(\alpha)$ occurs at every change of direction; in Fig. 4b, the deviation $(\alpha)$ is 56 degrees; in Fig. $4 c$, the deviation is zero degrees. Note that the straightening, progressing from Fig. 4 a to Fig. 4b (see Fig. 4 caption), has important consequences on the figural organization. From an initial state in which we perceive only diamonds against a background, we pass to a 3-D organization in which diamonds and squares appear as facets of a polyhedral surface (corrugation effect), and finally to a grid of orthogonal lines. (See Supplementary Material 2 for a more finely graded progression of straightening of the lines.)

\section{Conclusions from Figs. 3 and 4}

The effects of straightness manipulation are clearly visible. They cause the transition in Fig. 3 from a figural organization

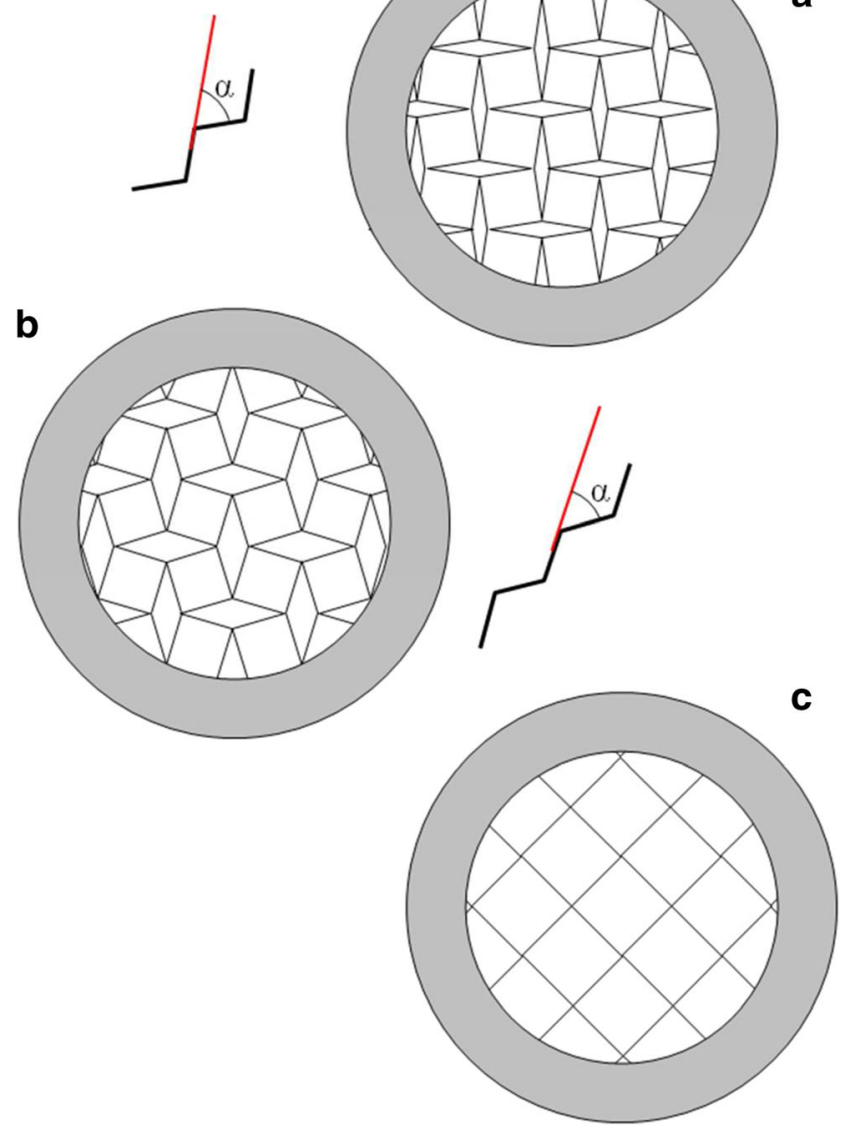

Fig. 4 Perceptual result of jagged lines crossing at inflection points. The lines embedded in the circle are three examples of intersecting lines of different linearity: jagged lines (a), less jagged lines (b), straight lines (c). The two upper figures are flanked by an enlargement of a portion of the figure to illustrate the deviation from straightness $(\alpha)$. (Color figure online)

in which the stars emerge as figures to a different organization in which we see crossing bundles of lines. The organizations are more complex in Fig. 4. An initial configuration made up of diamonds transforms into a corrugated surface where diamonds and squares appear as facets of a polyhedral pattern; this pattern is replaced by intersecting bundles of lines when these come close to straightness.

In Fig. 3, the transition from one perceptual organization to another can be accounted for by the action of the closure and good continuation factors. When the short segments group to form closed contours, they obey to the closure rule. If a progressive decrease of $\alpha$ generates the appearances of jagged lines, then one can assume that the segments regrouping conforms to the good-continuation rule. Therefore, the transition can be considered the perceptual effect of a change in strength of the ratio between two grouping factors.

The same account can be put forward for the transitions illustrated in Fig. 4, but the appearance of the corrugated pattern raises the question of whether closure and good 
continuation interact in an unexplored way or new organizing factors emerge. The former is at the origin of the diamondsorganization of Fig. 4s, while the latter make the lines emerge in Fig. $4 \mathrm{c}$. In fact, their role is not easily recognizable when the corrugated surface emerges. The good continuation we speak of is not even an uncommon generalization (see, e.g., Kellman \& Shipley, 1991) of the classic Gestalt good continuation whereby the letter $X$ is perceived as two lines crossing rather than angles touching at a vertex.

They seem to act in synergy - the former to group the segments into the diamonds and squares contour, and the latter to form the folding lines of the polyhedral surface.

Here, three figural grouping factors seem to sum in their effects. If we choose one of the short segments of the jagged lines, we easily check that it belongs to three different figural organizations. It groups with three segments to form the contour of a diamond but with three different segments to form the contour of a square, and, finally, it joins to a series of identical segments to form a jagged folding line. Two of these groups are perceived as facets of a polyhedron, the third as a line of surface folding. Is the organization that emerges is the result of closure and good continuation balance? Is such a balance, if there is one, broken with a further straightening?

To answer these questions, we explored the conditions under which the strength of the two factors may be predicted.

\section{Predictions on the basis of the good-continuation rule}

In Fig. 5, a portion of patterns similar to Figs. $4 \mathrm{a}-\mathrm{b}$ is reproduced: two bundles (one red and the other blue) of jagged lines intersect forming a right angle (the axes of the jagged lines are orthogonal). A dotted line indicates the prolongation of a segment of the red line along the straight trajectory; $\alpha$ and $\beta$ indicate the angles that the prolongation forms with the two adjacent segments, one with the same line (red) the other with the intercepting line (blue). The width of these angles indicates the magnitude of the two possible deviations. This segment has two possible paths to follow: the red or the blue one. The good-continuation rule leads to the assumption that it will tend to prolong in the less deviating direction. The red dashed segment deviates $\beta$ degrees to group with the blue one, and $\alpha$ degrees to group with a red segment ( $\alpha$ is the straightness deviation). The good continuation leads us to predict different groupings depending on the width ratio between $\alpha$ and $\beta$. We can detail the predictions of what direction should prevail as follows.

$\alpha>\beta$ The good-continuation rule leads to predict that the red deviates and joins with the blue segment because this change of direction is smaller than the direction necessary to join to the adjacent red segment. This direction of grouping conforms to the closure rule as well. (This predicts what happens in Fig. $4 \mathrm{a}$, where diamonds are the predominant percept.)

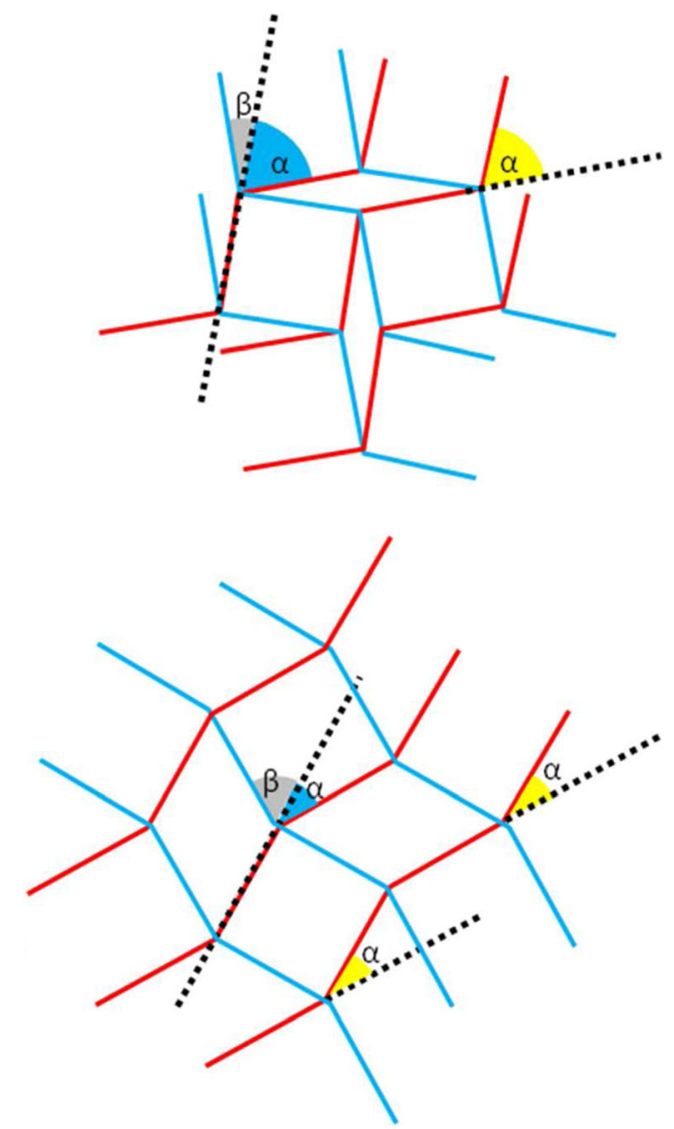

Fig. 5 Directions of line grouping following the good-continuation rule. Two intercepting jagged lines have been symbolized by red and blue lines. Dotted line indicates the prolongation of one of the red component segments; $\alpha$ and $\beta$ indicate the angles that the prolongation forms with the two adjacent segments, one with the same line (red) the other with the intercepting line (blue). The width of these angles indicates the magnitude of the two possible deviations. The red dashed segment deviates of $\beta$ degrees to group with the blue one, and of $\alpha$ degrees to group with a red segment ( $\alpha$ is the straightness deviation). "Good continuation" leads us to predict different groupings according to the width ratio between $\alpha$ and $\beta$. (Color figure online)

$\alpha=\beta$ In this configuration, the two angles are 45 degrees wide. The two laws of closure and good continuation balance and are assumed to have equal strength. With such conflicting solutions, we should expect an unstable percept: sometimes diamonds, some other times jagged lines. Fig. $4 \mathrm{~b}$ illustrates an alternative stable solution from the visual system: a corrugated surface. This is a very subtle solution that renders the two laws again allied to generate local closed contours and global dividing lines.

$\alpha<\beta$ This is the condition of rivalry between the closure law and the good-continuation laws. In fact, the dotted segment is expected to join to the smaller deviating segment, the red one, a grouping that is in opposite direction to the one determined by closure. Here, we predict the vanishing of diamonds and the appearance of jagged lines analogous to the phenomenon seen in Fig. 3c. We have done a study to test these predictions. 
1. To calculate, by a psychophysical procedure, the values of $\alpha$ associated to the transition from a figural organization to a different one, in particular from isolated figures to corrugated surface and from isolated figures to bundles of lines.

2. To check the influence of the spatial extension of stimuli. We assumed that if global organizing factors are at the origin of figural organizations, then they should manifest stronger effects with stimuli sufficiently extended in space. For example, the threshold of appearance (in terms of $\alpha$ magnitude) and the persistence of the corrugation effect should be different with stimuli of different spatial extension.

\section{Method}

\section{Stimuli}

Two groups of stimuli were created. Group A consisted of jagged lines crossing at the midpoints between junctions (see Fig. 3). Group B consisted of jagged lines crossing at the folding points (see Fig. 4).

Group A Thirteen configurations (see Supplementary Material 1) were used with the values of $\alpha$ varied from a maximum of 62.13 degrees to a minimum of 4.77 in steps of 4.3 degrees.

Group B Nine configurations (see Supplementary Material 2) from the maximum value of $\alpha$ of 81.25 degrees to a minimum value of 55.25 degrees in steps of 3.25 degrees.

The stimuli were reproduced twice, with a large and a narrow spatial extent. They were displayed in a circular aperture surrounded by an annulus 14-cm wide. Two apertures were introduced, 3.8 degrees and 7.6 degrees in diameter for the narrow and the wider blocks, respectively.

Two blocks were made with the stimuli of Group A (starlike) consisting in 182 trials (13 configurations $\times 14$ presentations) and the other two were with the stimuli of the Group B (corrugated) consisting in 126 trials (9 configurations $\times 14$ presentations).

\section{Subjects}

Five right-handed voluntary students from the University of Padova (three female, two male, age range: $21-24$ years) and one of the experimenters (S.R.), with normal or corrected vision participated in the experiment. Participants were debriefed after the final session and received an explanation of the experiment. All subjects gave written informed consent and were treated in accordance with the ethical guidelines of the Department of General Psychology and according to the principles expressed in the Declaration of Helsinki. There were no adverse events or complaints. The subjects were shown some examples of the stimuli and instructed as follows:

"A figure such as this one will be displayed for half a second. Your task is to indicate whether you perceive only lines or bundles of crossing lines or something different, for example closed figures or surfaces (flat or corrugated)." Stimuli of the Group A were displayed, and the subject was instructed to choose between these alternative responses lines or stars. In case of uncertainty, the response could be randomly chosen. Then stimuli of the Group B were displayed, and the participant was asked to choose between two responses: diamonds (or squares) and corrugated surface.

\section{Apparatus, stimulus presentation and timing}

The participants were seated in a dimly lit room in front of a CRT color monitor $(1280 \times 1024$ pixels $)$ with a monitor retrace rate of $85 \mathrm{~Hz}$ at a viewing distance of approximately $75 \mathrm{~cm}$. Stimuli were generated with MATLAB Psychophysics Toolbox (Brainard, 1997; Pelli, 1997) and were presented using the psychophysical method of the constant stimuli (random presentation). Participants performed four blocks. Stimuli were displayed at the center of the screen for $500 \mathrm{~ms}$, and their entire size corresponded to $9 \times 9$ degree of visual angle (degrees). Participants responded with their left and right index fingers via a standard keyboard. The next trial started $1 \mathrm{sec}-$ ond after participant's response.

\section{Results}

Two measures were taken into consideration:

1. Stimuli A: The transition from stars to grid of lines was recorded

2. Stimuli B: The transition from diamonds to corrugate was recorded. The transition from corrugate to grid was uncertain and corresponds to very low $\alpha$ amplitudes; therefore, we decided to neglect it.

We fit our data, for each subject with a logistic function (Prins \& Kingdom, 2009). The four graphs in Fig. 6 show the corrugated and grid of lines percentages of responses of the subjects averaged and plotted as a function of deviation from straightness $(\alpha)$ (the functions in the figure were fitted to the mean data for illustrative purpose). The threshold (see Table 1) used corresponds to the value of $\alpha$, for which we obtained a $75 \%$ of response of corrugated or grid of lines. The precision is indicated by 

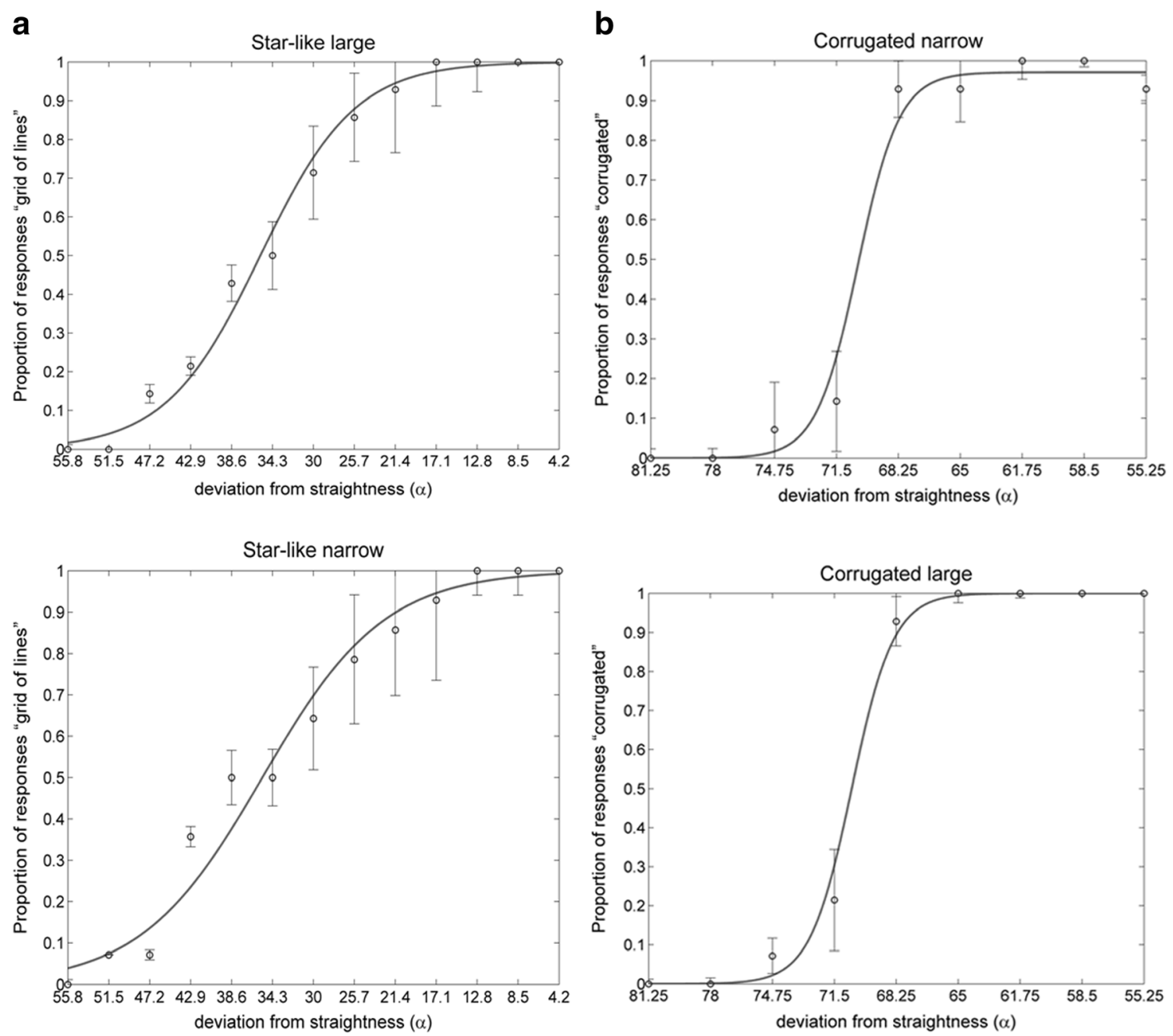

Fig. 6 Experimental results. The psychometric functions obtained for the four experimental conditions are shown. See text for discussion

the slope of the psychometric function (a higher value indicates more precision).

A series of paired $t$ tests between corrugated blocks (large vs. narrow) and star-like blocks (large vs. narrow) were performed to investigate whether the aperture could change the phenomenological appearance of the configuration. No difference was found in the threshold, slope, or asymptotes, indicating that the size of the aperture did not matter.

Table 1 Thresholds for the value of $\alpha$, for which we obtained $75 \%$ of response of grid of lines (Stimuli A) or corrugated (Stimuli B)

\begin{tabular}{llll}
\hline & Thresholds $(\mathrm{deg})$ & Slope & Upper asymptote \\
\hline Star-like large & $31.2(S D=7.8)$ & 1.26 & 0.99 \\
Star-like narrow & $34.6(S D=11.8)$ & 3.85 & 0.9 \\
Corrugated large & $69.5(S D=2.1)$ & 7.66 & 1.0 \\
Corrugated narrow & $70.1(S D=3.9)$ & 7.43 & 0.96 \\
\hline
\end{tabular}

\section{Discussion}

In the first experiment, subjects had to choose between describing their perception as either stars or lines with the star perception being interpreted as being caused by the Gestalt closure rule. Convex octagons can also be seen in these figures. Along with the general utility of a two-alternative forcedchoice design, we did not include octagon as a choice, as, in our own perception of the figures, we found this not to be a predominant percept. However, experiments including this choice can be considered in the future.

The values found in this experiment for the switch points show different transitions from one organization to another. The corrugation effect arises suddenly with $\alpha$ mean magnitude close to 70 degrees, whereas the transition from the starlike organization and the grid of jagged lines is more gradual and progressive. The stimuli are different, and the judgment may follow different strategies, but it is doubtless that there is higher uncertainty in judging what organization emerges in Fig. 3. Whether closed contours (star-like shapes) or jagged 
lines are drawn is difficult to determine when the deviation from straightness is in the range $33 \pm 5$ degrees.

No effect of the spatial extension of stimuli was found. The regular and periodic pattern that may emerge only within a stimulus sufficiently extended in space are not involved as a structuring factor in the rise of the corrugation effect.

Good continuation and closure provide a good account of the findings for Fig. 3. The transition from one perceptual organization to a different one can be considered the result of these two competing rules varying in relative strength in relation to the jaggedness of the lines: The progressive variation of this parameter changes the relative strengths so that one of them prevails or they balance.

Without doubt, closure plays a role in figures like Fig. 4. However, the good-continuation hypothesis conflicts with two findings. First of all, the predicted direction of segment integration is not confirmed by the psychophysics results: In fact, the deviation of straightness associated with the appearance of the corrugation effect contrasts with the predictions that for $\alpha>$ 45 degrees the good continuation and the closure act in the same direction. Second, the hypothesized competition between good continuation and closure has different manifestations on the stimuli of Figure 3 and Figure 4. The psychometric functions demonstrate a sudden transition from one figural organization to another in the former case and a gradual transition in the latter. (This transition zone may indicate an interesting possibly novel ambiguous, bistable percept.) The hypothesis is that other factors different from good continuation come into play when the diamonds are replaced by a corrugated surface.

We do not think other classic Gestalt principles can explain our results. The polyhedral configuration could be considered a Gestalt good form, but it is not clear why this form appears out of the bundle of lines. Thus, from the results, a main problem arises: Why does the transitional state appear as a polyhedral corrugation - that is, why a 3-D figural organization and not tiling or grid of lines? Extensive literature on machine vision reports numerous attempts to derive a 3-D representation of polyhedra from line drawings: pioneering works date decades ago (Barrow \& Tenenbaum, 1978; Clowes, 1971; Huffman, 1971; Kanade, 1981; Stevens, 1981; Sugihara, 1986) and were followed by intense research (for more recent surveys, see reviews by M. Cooper, 2008; Johnson, Gross, Hong, \& Do, 2009). Research is based on the assumption that line drawings that depict the edges and vertices of objects provide perceptually salient information about 3-D shape. Computer programs that achieve the interpretation of line drawings as polyhedral scenes (without the recourse to particular object prototypes) have been proposed. One of the initial crucial steps consists in finding algorithms able to label the line drawings, that is, to interpret a line as convex, concave or contour line (M. C. Cooper, 2007; Mardzuki \& Haron, 2008; P. Varley, 2005, 2009). Initial attempts were successful. The Clowes-Huffman catalogue for line drawings of trihedral polyhedra is recognized as a well-established contribution: Nevertheless, the problems are much more difficult with tetrahedral polyhedral patterns (P. A. C. Varley \& Martin, 2001).

Some questions remain unanswered and require deeper understanding of the laws of perceptual organization. The first concerns the relation between the angles of figure contours and the likelihood that these contours reassemble to form lines or different contours. We have demonstrated that sharp angles lead to perceived diamonds, but the reason that a widening beyond a threshold of these angles give rise to a new organization is unknown. The second issue deals with the relation between shape regularity and polyhedral organization. The corrugation perception can be elicited by irregular shapes (wrinkled paper, for example). It should be interesting to explore whether a template of corrugation is a condition for the polyhedral perceptual organization as well as generally the ability of the visual system to solve the ambiguity in the interpretation of line drawings.

\section{Conclusion}

In light of the demonstrations and the results here reported we have to conclude that the perceptual groupings may give origin to figural organizations that merit a careful investigation. The lines organize into polyhedral surfaces despite the violation of good-continuation rule and consist in a structure of such complexity to challenge algorithms of computer vision and stimulate a deeper understanding of the perceptual interpretation of groups of lines - perhaps as 3-D structures. To have an idea of the ability of the visual system to generate these structures in an instant of time it is sufficient to consider that a 3-D polyhedral surface suddenly reverses into one of three possible structures in which the concavity of edges reverses to convexity and vice versa. The figures we have studied here present a real challenge to our knowledge of perceptual organization.

\section{References}

Barrow, H., \& Tenenbaum, J. (1978). Recovering intrinsic scene characteristics. In A. Hanson \& E. Riseman (Eds.), Computer vision systems (pp. 3-26). New York: Academic Press.

Beck, J., Rosenfeld, A., \& Ivry, R. (1989). Line segregation. Spatial Vision, 4, 75-101.

Brainard, D. H. (1997). The psychophysics toolbox. Spatial Vision, 10(4), 433-436.

Clowes, M. B. (1971). On seeing things. Artificial Intelligence, 2(1), $79-116$.

Cooper, M. (2008). Line drawing interpretation. Berlin: Springer.

Cooper, M. C. (2007). Constraints between distant lines in the labelling of line drawings of polyhedral scenes. International Journal of Computer Vision, 73(2), 195-212. 
Feldman, J. (1996). Regularity vs genericity in the perception of collinearity title. Perception, 25, 335-342.

Feldman, J. (1997). Curvilinearity, covariance, and regularity in perceptual groups. Vision Research, 37, 2835-2848.

Glass, L., \& Perez, R. (1973). Perception of random dot interference patterns. Nature, 246, 360-362.

Grünbaum, B., \& Shephard, G. C. (1987). Tilings and patterns. New York: Freeman.

Huffman, D. A. (1971). Impossible objects as nonsense sentences. Machine Intelligence, 6(1), 295-323.

Johansson, G. (1975). Visual motion perception. Scientific American, 232, 76-88.

Johnson, G., Gross, M. D., Hong, J., \& Do, E. Y. (2009). Computational support for sketching in design: A review. Foundations and Trends in Human-Computer Interaction, 2(1), 1-93.

Kanade, T. (1981). Recovery of the three-dimensional shape of an object from a single view. Artificial Intelligence, 17, 409-460.

Kellman, P. J., \& Shipley, T. F. (1991). A theory of visual interpolation in object perception. Cognitive Psychology, 23(2), 141-221.

Mardzuki, S., \& Haron, H. (2008). Interpretation of drawing: A review on line labeling. The 4th Postgraduate Annual Research Seminar (PARS08), 30, 247-250.

Marroquin, J. L. (1976). Human visual perception of structure (Master's thesis, Massachusetts Institute of Technology, Cambridge, MA).

Pelli, D. G. (1997). Pixel independence: Measuring spatial interactions on a CRT display. Spatial Vision, 10, 443-446.

Pinna, B. (2010). What comes before psychophysics? The problem of 'what we perceive' and the phenomenological exploration of new effects. Seeing and Perceiving, 23, 463-481.

Prins, N., \& Kingdom, F. A. A. (2009). Palamedes: Matlab routines for analyzing psychophysical data. Retrieved from http://www. palamedestoolbox.org

Smits, J. T. S., Vos, P. G., \& Van Oeffelen, M. P. (1985). The perception of a dotted line in noise: A model of good continuation and some experimental results. Spatial Vision, 1, 163-177.
Stevens, K. A. (1981). The visual interpretation of surface contours. Artificial Intelligence, 17(1/3), 47-73.

Sugihara, K. (1986). Machine interpretation of line drawings (Vol. 1). Cambridge: MIT Press.

Uttal, W. R. (1973). The effect of deviations from linearity on the detection of dotted line patterns. Vision Research, 13, 2155-2163.

Uttal, W. R., Bunnell, L., \& Corwin, S. (1970). On the detectability of straight lines in visual noise: An extension of French's paradigm into the millisecond domain. Perception \& Psychophysics, 8, 385-388.

van der Helm, P. A. (2014). Symmetry perception. In J. Wagemans (Ed.), Oxford handbook of perceptual organization. Oxford: Oxford University Press.

Varley, P. (2005). Extended vertices: A problem for line labelling. Proceedings of the Digital Engineering Workshop: 5th JapanKorea CAD/CAM Workshop (pp. 106-114).

Varley, P. (2009). Problems for line labelling: A test set of drawings of objects with higher-valency vertices. International Journal of $C A D$ / CAM, 5(1). Available from http://www.ijcc.org/ojs/index.php/ijcc/ article/view/37

Varley, P. A. C., \& Martin, R. R. (2001). The junction catalogue for labelling line drawings of polyhedra with tetrahedral vertices. International Journal of Shape Modeling, 7(1), 23-44.

Wagemans, J. (1993). Skewed symmetry: A nonaccidental property used to perceive visual forms. Journal of Experimental Psychology. Human Perception and Performance, 19, 364-380.

Wagemans, J., Elder, J. H., Kubovy, M., Palmer, S. E., Peterson, M. A., Singh, M., \& von der Heydt, R. (2012). A century of Gestalt psychology in visual perception: I. Perceptual grouping and figureground organization. Psychological Bulletin, 138(6), 1172.

Wertheimer, M. (1923). Untersuchungen zur Lehre von der Gestalt, II. Psychologische Forschung, 4, 301-350. In W. D. Ellis (Ed.), (Translated extract reprinted as "Laws of organization in perceptual forms," in A source book of Gestalt psychology (pp. 71-94). London: Routledge \& Kegan Paul Ltd.

Wertheimer, M., \& Spillmann, L. (2012). On perceived motion and figural organization. Cambridge: MIT Press. 